\title{
Online Sum-Paintability: Slow-Coloring of Trees
}

\author{
Gregory J. Puleo and Douglas B. West
}

March 25, 2022

\begin{abstract}
The slow-coloring game is played by Lister and Painter on a graph $G$. On each round, Lister marks a nonempty subset $M$ of the remaining vertices, scoring $|M|$ points. Painter then gives a color to a subset of $M$ that is independent in $G$. The game ends when all vertices are colored. Painter's goal is to minimize the total score; Lister seeks to maximize it. The score that each player can guarantee doing no worse than is the sum-color cost of $G$, written $\stackrel{\circ}{\mathrm{s}}(G)$. We develop a linear-time algorithm to compute $\stackrel{\circ}{\mathrm{s}}(G)$ when $G$ is a tree, enabling us to characterize the $n$-vertex trees with the largest and smallest values. Our algorithm also computes on trees the interactive sum choice number, a parameter recently introduced by Bonamy and Meeks.
\end{abstract}

Keywords: slow-coloring game; tree; stem vertex; interactive sum choice number

\section{Introduction}

The slow-coloring game (introduced in [10]) models the difficulty of producing a proper coloring of a graph $G$ when it is not known in advance which vertices are allowed to have which colors. The players are Lister and Painter. On the $i$ th round, Lister marks a nonempty subset $M$ of the uncolored vertices, scoring $|M|$ points. Painter gives color $i$ to a subset of $M$ that is independent in $G$. The game ends when all vertices are colored. Painter wants to minimize the total score; Lister wants to maximize it. The score that each player can guarantee achieving is the sum-color cost of $G$, written $\stackrel{\circ}{\mathrm{s}}(G)$.

This game is an online version of the "painting game", which is an online version of list coloring. List coloring generalizes classical graph coloring by introducing a list assignment $L$ that assigns to each vertex $v$ a set $L(v)$ of available colors. A graph $G$ is $L$-colorable if it has a proper coloring $\phi$ with $\phi(v) \in L(v)$ for every vertex $v$. Given $f: V(G) \rightarrow \mathbb{N}$, a graph $G$ is $f$-choosable if $G$ is $L$-colorable whenever $|L(v)| \geq f(v)$ for all $v$.

Introduced by Vizing [13] and by Erdös, Rubin, and Taylor [4], the choosability of a graph $G$ is the least $k$ such that $G$ is $f$-choosable whenever $f(v) \geq k$ for all $v \in V(G)$. Alternatively, we may minimize the sum (or average) of list sizes. Introduced by Isaak [8, 9] and studied also in [1, 6, 7, 11], the sum-choosability of a graph $G$, denoted $\chi_{\mathrm{SC}}(G)$, is the minimum of $\sum f(v)$ when $G$ is $f$-choosable. 
In the $f$-painting game, the color lists are not known in advance. In round $i$, Lister marks a set $M$ of vertices allowed to receive color $i$; this can represent the set of vertices having color $i$ in their lists. Painter chooses an independent subset of $M$ to receive color $i$. Lister can design later marked sets based on Painter's choices. Lister wins if some vertex is marked more than $f(v)$ times; Painter wins by first coloring all the vertices. The graph is $f$-paintable if Painter has a winning strategy. Introduced by Schauz [12] and by Zhu [14], the paintability is the least $k$ such that $G$ is $f$-paintable whenever $f(v) \geq k$ for all $v \in V(G)$. Introduced by Carraher et al. [3] and studied also in [11], the sum-paintability of a graph $G$, denoted $\chi_{\mathrm{SP}}(G)$, is the least value of $\sum f(v)$ for a function $f$ such that $G$ is $f$-paintable. Since Lister marks sets in response to Painter's choices, $\chi_{\mathrm{SP}}(G) \geq \chi_{\mathrm{SC}}(G)$ for all $G$.

We view $f$ as allocating tokens to vertices; marking a vertex uses up a token. Compared to sum-paintability, the slow-coloring game gives some help to Painter by allowing Painter to postpone allocating tokens to vertices until they are needed. The sum-color cost $\stackrel{\mathrm{S}}{(} G)$ equals the minimum number of tokens Painter must have available to guarantee producing a coloring. Since Painter can always play as if the available tokens are given by a function $f$ such that $G$ is $f$-paintable, $\stackrel{\circ}{\mathrm{s}}(G) \leq \chi_{\mathrm{SP}}(G)$.

Mahoney, Puleo, and West [10] proved various results on $\stackrel{\circ}{\mathrm{s}}(G)$. With $\alpha(G)$ denoting the independence number, always $\frac{|V(G)|}{2 \alpha(G)}+\frac{1}{2} \leq \frac{\stackrel{\varsigma}{\varsigma}(G)}{|V(G)|} \leq \max \left\{\frac{|V(H)|}{\alpha(H)}: H \subset G\right\}$. Equality holds in $\stackrel{\circ}{\mathrm{s}}(G) \leq \chi_{\mathrm{SP}}(G)$ if and only if all components are complete. For complete bipartite graphs, $r+\frac{5}{2} s-3+\sqrt{2 r-2 s}<\stackrel{\circ}{\mathrm{s}}\left(K_{r, s}\right) \leq r+s+2 \sqrt{r s}$ when $r \geq s$, with $\stackrel{\circ}{\mathrm{s}}\left(K_{r, r}\right) \sim 4 r$ conjectured.

In this paper, we extend their results on trees. For $k, r \in \mathbb{N}$, let $t_{k}=\left(\begin{array}{c}k+1 \\ 2\end{array}\right)$ and $u_{r}=$ $\max \left\{k: t_{k} \leq r\right\}$. The numbers $t_{k}$ are the triangular numbers. Note that $u_{r}=\left\lfloor\frac{-1+\sqrt{1+8 r}}{2}\right\rfloor$.

Theorem 1.1 ([10]). For every $n$-vertex tree $T$,

$$
n+\sqrt{2 n} \approx n+u_{n-1}=\stackrel{\circ}{\mathrm{s}}\left(K_{1, n-1}\right) \leq \stackrel{\circ}{\mathrm{s}}(T) \leq \stackrel{\circ}{\mathrm{s}}\left(P_{n}\right)=\lfloor 3 n / 2\rfloor .
$$

Let a stem in a forest be a vertex having a leaf neighbor and at most one non-leaf neighbor. We now state our main result.

Theorem 1.2 (Main Theorem). Let $T$ be a forest. If $T$ has no edges, then $\stackrel{\mathrm{s}}{ }(T)=|V(T)|$. If $v$ is a stem in $T$ and $R$ is the set of leaf neighbors of $v$, with $r=|R|$, then

$$
\stackrel{\mathrm{s}}{ }(T)= \begin{cases}\stackrel{\circ}{\mathrm{s}}(T-R-v)+r+1+u_{r}, & \text { if } r+1 \text { is not a triangular number }, \\ \stackrel{\mathrm{s}}{\mathrm{s}}(T-R)+r+u_{r}, & \text { if } r+1 \text { is a triangular number. }\end{cases}
$$

Since stems are easy to find (the neighbor of a leaf on a longest path is a stem), this result gives a linear-time algorithm to compute $\stackrel{\circ}{\mathrm{s}}$ on forests. We also use it to characterize the extremal trees.

Theorem 1.3. If $T$ is an n-vertex forest, then $\stackrel{\mathrm{s}}{\mathrm{S}}(T)=\lfloor 3 n / 2\rfloor$ (the maximum) if and only if $T$ contains a spanning forest in which every vertex has degree 1 or 3 , except for one vertex of degree 0 or 6 when $n$ is odd. If $n \geq 4$ and neither $n-1$ nor $n-2$ is a triangular number, then $\stackrel{\mathrm{s}}{\mathrm{S}}(T)=n+u_{n-1}$ (the minimum) if and only if $T$ is a star (in the remaining cases, $a$ few additional trees achieve the minimum). 
Further results have been obtained by Gutowski, Krawczyk, West, Zajac, and Zhu [5]. Let $G$ be an $n$-vertex graph. Always $\stackrel{\circ}{(} G) \leq k n$ when $G$ is $k$-colorable, because Painter can always color at least $|M| / r$ vertices when $M$ is marked. Hence $\stackrel{\circ}{\mathrm{s}}(G) \leq(1+d) n$ when $G$ is $d$-degenerate; [5] proves $\stackrel{\mathrm{s}}{ }(G) \leq\left(1+\frac{3}{4} d\right) n$ (the bound must be at least $\left(1+\frac{1}{2} d\right) n$, by $\left.\frac{n}{d+1} K_{d+1}\right)$. Outerplanar graphs are 2-degenerate, but here [5] improves the bound to $\stackrel{\mathrm{s}}{\mathrm{S}}(G) \leq \frac{7}{3} n$ (the

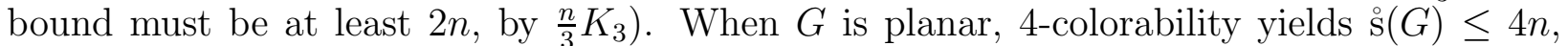
but [5] improves the bound to $\stackrel{\circ}{s}(G) \leq 3.9857 n$ (the bound must be at least $\frac{5}{2} n$, by $\frac{n}{4} K_{4}$ ).

A related parameter called the interactive sum choice number was introduced by Bonamy and Meeks [2]. Consider the following game between two players, whom we call Requester and Supplier. Initially, each vertex has an empty color list $L(v)$. In each round of the game, Requester selects a vertex $v$ and requests a new color for its list; Supplier chooses a color not already present in $L(v)$ and adds it to the list. The game continues until the lists $L$ are such that $G$ is $L$-colorable. Requester's goal is to minimize the total number of requests (rounds), while Supplier's goal is to maximize it. The interactive sum choice number of $G$, written $\chi_{\mathrm{ISC}}(G)$, is the common value that both players can guarantee.

The interactive sum choice game is similar to the slow-coloring game, with Requester analogous to Painter and Supplier analogous to Lister. The games differ in who starts each round: in slow-coloring, Painter responds to Lister, while in interactive sum choice Supplier responds to Requester. Bonamy and Meeks posed the problem of computing $\chi_{\mathrm{ISC}}(T)$ when $T$ is a forest, giving a formula for the value on stars, which we state in our notation.

Theorem 1.4 (Bonamy-Meeks [2]). $\chi_{\mathrm{ISC}}\left(K_{1, r}\right)=r+1+u_{r}$.

The formula in Theorem 1.4 agrees with the formula for $\stackrel{\mathrm{s}}{(}\left(K_{1, n-1}\right)$ in Theorem 1.1, so $\chi_{\mathrm{ISC}}(T)=\stackrel{\circ}{\mathrm{s}}(T)$ when $T$ is a star. In Section 5 , we prove $\chi_{\mathrm{ISC}}(T)=\stackrel{\circ}{\mathrm{s}}(T)$ for every forest $T$. Thus Theorem 1.2 also provides an algorithm to compute $\chi_{\mathrm{ISC}}(T)$ on forests.

Bonamy and Meeks proved $\chi_{\mathrm{ISC}}\left(C_{n}\right)=3 n / 2+1$ for the $n$-cycle $C_{n}$ with $n$ even, but one can obtain $\stackrel{\circ}{\mathrm{s}}\left(C_{n}\right)=\lceil 3 n / 2\rceil$ using the value of $\stackrel{\mathrm{s}}{\mathrm{s}}\left(P_{n}\right)$; thus $\stackrel{\mathrm{s}}{\mathrm{S}}\left(C_{2 k}\right)<\chi_{\mathrm{ISC}}\left(C_{2} k\right)$. Bonamy and Meeks also noted that $\chi_{\mathrm{ISC}}(G) \leq \chi_{\mathrm{SC}}(G)$ for all $G$, since Requester can achieve list sizes $f$ such that $G$ is $f$-choosable; we ask whether there are graphs with $\stackrel{\circ}{(}(G)>\chi_{\mathrm{SC}}(G)$.

\section{Basic Observations}

Unlike sum-paintability, sum-color cost is given by an easily described (but hard to compute) recursive formula. The key point is that prior choices do not affect Painter's optimal strategy for coloring subsets of marked sets on the remaining subgraph.

Proposition 2.1 ([10]). $\quad \stackrel{\circ}{\mathrm{s}}(G)=\max _{\varnothing \neq M \subseteq V(G)}(|M|+\min \stackrel{\circ}{\mathrm{s}}(G-I))$, where the minimum is taken over subsets I of $M$ that are independent in $G$.

Proof. In response to any initial marked set $M$, Painter chooses an independent subset $I \subseteq M$ to minimize the cost of the remainder of the game. 
In studying optimal strategies for Lister and Painter, not all legal moves need be considered. Let $G[S]$ denote the subgraph of $G$ induced by a set $S \subseteq V(G)$.

Observation 2.2 ([10]). On any graph, there are optimal strategies for Lister and Painter such that Lister always marks a set $M$ inducing a connected subgraph $G[M]$, and Painter always colors a maximal independent subset of $M$.

Proof. A move in which Lister marks a disconnected set $M$ can be replaced with successive moves marking the vertex sets of the components of $G[M]$. For the second statement, coloring extra vertices at no extra cost cannot hurt Painter.

Other easy observations yield useful bounds. The lower bound below was observed in [10]. Let $[A, B]$ denote the set of edges with one endpoint in $A$ and one endpoint in $B$.

Lemma 2.3. If $G$ is a graph and $(A, B)$ is a bipartition of $V(G)$, then

$$
\stackrel{\circ}{\mathrm{s}}(G[A])+\stackrel{\circ}{\mathrm{s}}(G[B]) \leq \stackrel{\circ}{\mathrm{s}}(G) \leq \stackrel{\circ}{\mathrm{s}}(G[A])+\stackrel{\circ}{\mathrm{s}}(G[B])+|[A, B]| .
$$

Proof. For the lower bound, Lister can play an optimal strategy on $G[A]$ while ignoring the rest and then do the same on $G[B]$, achieving the score $\stackrel{\circ}{\mathrm{s}}(G[A])+\stackrel{\circ}{\mathrm{s}}(G[B])$.

For the upper bound, Painter uses optimal strategies on $G[A]$ and $G[B]$. When doing so requests coloring of both endpoints of an edge in $[A, B]$, Painter allocates an extra token to each such endpoint $v$ in $B$ and instead makes the optimal response in $B$ to the marked set obtained by omitting those vertices from the actual marked set. Each edge of the cut acts in this way at most once, because when it does the endpoint in $A$ is colored.

If $T_{1}, \ldots, T_{k}$ are trees such that $\left|V\left(T_{i}\right)\right|=n_{i}$ and $\stackrel{\circ}{\mathrm{s}}\left(T_{i}\right)=3 n_{i} / 2$, then the disjoint union of $T_{1}, \ldots, T_{k}$ is a forest $F$ with $\stackrel{\circ}{\mathrm{S}}(F)=3|V(F)| / 2$, by the lower bound in Lemma 2.3. Adding edges to turn $F$ into an $n$-vertex tree $T$ does not reduce the sum-color cost, so $\stackrel{\circ}{\mathrm{S}}(T)=3 n / 2$, by Theorem 1.1. Thus there is a huge variety of trees achieving the maximum. Nevertheless, Theorem 1.3 states a simple structural characterization.

Our algorithm to compute $\stackrel{\circ}{S}$ on trees combines the bounds in Lemma 2.3 with an understanding of slow-coloring on stars. By Theorem 1.1, $\stackrel{\mathrm{s}}{\mathrm{S}}\left(K_{1, r}\right)=r+1+u_{r}$. Therefore, when $r+1$ is not a triangular number, the statement of Theorem 1.2 is $\stackrel{\circ}{\mathrm{s}}(T)=\stackrel{\mathrm{s}}{\mathrm{s}}(T[A])+\stackrel{\circ}{\mathrm{s}}(T[B])$, where $B=R \cup\{v\}$ and $A=V(T)-B$. There is only one edge joining $A$ and $B$, so proving this case only requires saving 1 in the upper bound from Lemma 2.3 .

Doing this requires a closer look at optimal play on a star. The claim we need includes a computation of $\stackrel{\circ}{\mathrm{S}}\left(K_{1, r}\right)$, making our presentation self-contained. In [10], $\stackrel{\circ}{\mathrm{S}}\left(K_{1, r}\right)$ was computed as a special case of the join of a complete graph with an independent set. Our argument for the special case is somewhat simpler and gives additional information about what moves are optimal. Recall that $u_{r}=\max \left\{k: t_{k} \leq r\right\}$, where $t_{k}=\left(\begin{array}{c}k+1 \\ 2\end{array}\right)$.

Lemma $2.4([10]) \cdot u_{r-u_{r}}=u_{r}$ when $r+1$ is triangular, and otherwise $u_{r-u_{r}}=u_{r}-1$.

Proof. If $u_{r}=k$, then $t_{k} \leq r<t_{k+1}$. Also $t_{k+1}-t_{k}=k+1$. Thus $r-k=t_{k}$ if $r+1=t_{k+1}$, yielding $u_{r-u_{r}}=u_{r}$. Otherwise $t_{k-1}<r-k<t_{k}$, which yields $u_{r-u_{r}}=u_{r}-1$. 
Theorem 2.5. $\stackrel{\circ}{\mathrm{s}}\left(K_{1, r}\right)=r+1+u_{r}$. Any optimal first move for Lister marks the center and $p$ leaves, where $u_{r} \leq p \leq u_{r}+r-t_{u_{r}}$, or marks only $p$ leaves, where $1 \leq p \leq r-t_{u_{r}}$. Painter can respond optimally by coloring the marked leaves, except that when $r+1$ is triangular and Lister marks the center and exactly $u_{r}$ leaves, the only optimal response for Painter is to color the center. Finally, if $r+1$ is not triangular and after one round of optimal play the uncolored subgraph is $K_{1, r^{\prime}}$, then again $r^{\prime}+1$ is not triangular.

Proof. We use induction on $r$, with basis $r=0$ using $u_{0}=0$. Suppose $r>0$.

If Lister marks $p$ leaves and not the center, then Painter colors all marked vertices. The score is then $p+\stackrel{\circ}{\mathrm{S}}\left(K_{1, r-p}\right)$, which by the induction hypothesis equals $r+1+u_{r-p}$. This equals the claimed value (and makes the Lister move optimal) if $u_{r-p}=u_{r}$. By monotonicity of $u$, this holds if and only $r-p \geq t_{u_{r}}$, and then $r-p+1$ is again not triangular.

Now suppose that Lister marks the center and $p$ leaves. Painter responds by coloring the center or all marked leaves. If the center is colored, then the score in the remainder of the game will be exactly $r$. Otherwise, the game continues on a star with $r-p$ leaves. Applying the recurrence of Proposition 2.1 and the induction hypothesis,

$$
\stackrel{\circ}{\mathrm{S}}\left(K_{1, r}\right)=\max _{p}\left[p+1+\min \left\{r, \stackrel{\circ}{\mathrm{S}}\left(K_{1, r-p}\right)\right\}\right]=r+1+\max _{p} \min \left\{p, 1+u_{r-p}\right\} .
$$

Since $u_{r-p}$ is a decreasing function of $p$, with $u_{r-p}>p$ when $p=0$ and $u_{r-p}<p$ when $p=r$, we seek $p$ such that $u_{r-p}=p-1$.

When $r+1$ is not triangular, setting $p=u_{r}$ yields $u_{r-p}=p-1$, by Lemma 2.4. Hence $p=u_{r}$ is optimal for Lister; Painter can color the center or the leaves. Smaller $p$ would yield smaller cost. For $j>0$, setting $p=u_{r}+j$ leads to cost $r+1+1+u_{r-p}$, which equals $r+1+u_{r}$ as long as $r-p \geq t_{u_{r}-1}$. Hence Lister can mark up to $u_{r}+\left(r-t_{u_{r}}\right)$ leaves, which Painter must color. When $p=u_{r}$, Painter may color either the center or the marked leaves.

In these cases, with Painter coloring leaves, the number $r^{\prime}$ of leaves remaining is $r-p$. We required $p \leq u_{r}+\left(r-t_{u_{r}}\right)$ to enforce $r-p \geq t_{u_{r}-1}$. Also, $r<t_{u_{r+1}}-1$ and $p \geq u_{r}$ yield $r-p<t_{u_{r+1}}-u_{r}-1=t_{u_{r}}-1$. Thus $t_{u_{r}-1} \leq r-p<t_{u_{r}}-1$, and $r^{\prime}+1$ is not triangular.

When $r+1$ is triangular, setting $p=u_{r}$ yields $p=u_{r-p}$ (by Lemma 2.4), and Painter must color the center. However, in this case also $1+u_{r-u_{r}-1}=u_{r-u_{r}}=u_{r}$. Thus setting $p$ to be $u_{r}+1$ again yields $\min \left\{p, 1+u_{r-p}\right\}=u_{r}$, but now Painter must color the leaves to respond optimally. As in the previous case, Lister can mark as many as $u_{r}+\left(r-t_{u_{r}}\right)$ leaves, which in this case equals $2 u_{r}$. Still Painter must color the leaves.

\section{Main Result}

When $T$ is a forest of stars, the statement of our main theorem (Theorem 1.2) reduces to the value given in Theorem 2.5 for stars. Note that the basis for the main theorem, $\stackrel{\circ}{\mathrm{S}}(T)=|V(T)|$ when $T$ has no edges, includes the case of the null graph, $|V(T)|=0$.

When $T$ has a component that is not a star, the computation in Theorem 1.2 tells us to break off a star having only one nonleaf neighbor. The center of such a star is a stem. Such 
vertices have sometimes been called "penultimate" vertices, but a stem need not be adjacent to an endpoint of a longest path.

The cases in proving our main theorem (Theorem 1.2) are based on Lemma 2.4. Throughout this section, $v$ is a stem in a forest $T$, the set of leaf neighbors of $v$ is $R$, and $r=|R| \geq 1$. The analysis is easy when $r+1$ is not triangular.

Lemma 3.1. If $r+1$ is not triangular, then $\stackrel{\circ}{\mathrm{s}}(T)=\stackrel{\circ}{\mathrm{s}}(T-R-v)+\stackrel{\circ}{\mathrm{S}}\left(K_{1, r}\right)$.

Proof. With $B=R \cup\{v\}$ and $A=V(T)-B$, we have $T[B] \cong K_{1, r}$, and the lower bound in Lemma 2.3 yields $\stackrel{\circ}{\mathrm{s}}(T) \geq \stackrel{\circ}{\mathrm{s}}(T-R-v)+\stackrel{\circ}{\mathrm{s}}\left(K_{1, r}\right)$. If $N(v) \cap A=\varnothing$, then the upper bound in Lemma 2.3 yields the desired equality, so we may assume $v$ has a neighbor in $A$.

For the upper bound, we use induction on $r$. When $r=1$, let $w$ and $z$ be the neighbors of $v$ in $A$ and $B$, respectively. Let $M$ be the first move by Lister. We gain the needed 1 over the upper bound in Lemma 2.3 unless $M \cap A$ is an optimal first move in $T[A]$ and $M \cap B$ is an optimal first move in $T[B]$. Hence Lister marks both vertices of $B$, and an optimal response by Painter in $T[B]$ colors one of them. Let $S$ be an optimal response for Painter to $M \cap A$. If $w \in S$, then Painter colors $S \cup\{z\}$. If $w \notin S$, then Painter colors $S \cup\{v\}$. In either case, the edge $w v$ is gone, and we never allocate an extra token for it, so optimal play in $A$ and $B$ separately continues, yielding the desired upper bound.

When $r>1$ and $r+1$ is not triangular, Painter has an optimal response to optimal play by Lister on $T[A]$ and $T[B]$ separately that colors only leaves from $T[B]$. Hence Painter can make this response, leaving $T\left[A^{\prime} \cup B^{\prime}\right]$, where $A^{\prime} \subseteq A$ and $B^{\prime} \subseteq B$. By Lemma 2.4 $T\left[B^{\prime}\right]$ is a star with center $v$ and $r^{\prime}$ leaves, where $r^{\prime}+1$ is not triangular. By the induction hypothesis, Painter has a strategy to complete the game with additional cost at most $\left.\stackrel{\mathrm{s}}{(} T\left[A^{\prime}\right]\right)+\stackrel{\circ}{\mathrm{s}}\left(K_{1, r^{\prime}}\right)$. Since the initial round was also by optimal play, the total cost is at most $\stackrel{\circ}{\mathrm{s}}(T[A])+\stackrel{\circ}{\mathrm{S}}\left(K_{1, r}\right)$.

Lemma 3.2. Always $\stackrel{\mathrm{s}}{\mathrm{S}}(T) \leq \stackrel{\circ}{\mathrm{s}}(T-R)+r+u_{r}$.

Proof. We use induction on $|V(T)|$. When $v$ is a central vertex of a star component with at least two vertices, the bound holds with equality, by Theorem 2.5, so the claim holds for all forests with such components. Hence we may assume that $v$ has a non-leaf neighbor and that the inequality holds for stems in all trees with fewer vertices.

By Lemma 2.3, $\stackrel{\circ}{\mathrm{s}}(T-R-v)+r+1+u_{r} \leq \stackrel{\circ}{\mathrm{s}}(T) \leq \stackrel{\circ}{\mathrm{s}}(T-R-v)+r+u_{r}+2$. Lemma 3.1 yields $\stackrel{\circ}{\mathrm{s}}(T)=\stackrel{\mathrm{s}}{\mathrm{s}}(T-R-v)+r+1+u_{r}$ when $r+1$ is not triangular. By Lemma 2.3, $\stackrel{\circ}{\mathrm{s}}(T-R) \geq \stackrel{\mathrm{s}}{\mathrm{s}}(T-R-v)+1$, and hence $\stackrel{\circ}{\mathrm{s}}(T) \leq \stackrel{\circ}{\mathrm{s}}(T-R)+r+u_{r}$ when $\stackrel{\mathrm{s}}{ }(T)=\stackrel{\mathrm{s}}{ }(T-R-v)+r+1+u_{r}$, such as when $r+1$ is not triangular. When $r+1$ is triangular, we give a strategy for Painter, using the induction hypothesis.

If the desired inequality does not hold, then $\stackrel{\mathrm{s}}{\mathrm{s}}(T)=\stackrel{\mathrm{s}}{\mathrm{s}}(T-R-v)+\stackrel{\circ}{\mathrm{s}}\left(K_{1, r}\right)+1$. Let $M$ be an optimal initial set marked by Lister; the restrictions of $M$ to $T-R-v$ and $T[R \cup\{v\}]$ must both be optimal first moves. Let $p=|M \cap R|$, and let $T^{\prime}=T-R$.

Case 1: $v \notin M$. Since $r+1=t_{u_{r}+1}$, we have $r-u_{r}=t_{u_{r}}$. We saw in Theorem 2.5 that marking $p$ leaves and not the center is optimal for Lister on $K_{1, r}$ only when $p \leq r-t_{u_{r}}=u_{r}$. Let $X^{\prime}$ be an optimal reply of Painter to the move $M-R$ on the subtree $T-R-v$, and let $X=X^{\prime} \cup(R \cap M)$. Painter colors $X$. 
In the remaining forest $T-X$, still $v$ is a stem and $v$ has $r-p$ leaf neighbors. Since $p \leq u_{r}$, the value $r-p+1$ is not triangular, and $u_{r-p}=u_{r}$. Hence Lemma 3.1 applies, and $\stackrel{\circ}{\mathrm{s}}(T-X)=\stackrel{\circ}{\mathrm{s}}(T-X-R-v)+(r-p)+u_{r-p}+1$. Thus, the optimal total score is $p+|M-R|+\stackrel{\circ}{\mathrm{s}}(T-X)$, which equals $\stackrel{\circ}{\mathrm{s}}(T-X-R-v)+r+u_{r-p}+1+|M-R|$. Since $X^{\prime}$ is an optimal response to the marked set $M-R$ on $T-R-v$ (and $T-X-R-v=T-X^{\prime}-R-v$ ), we have $\stackrel{\circ}{\mathrm{s}}(T-X-R-v)+|M-R| \leq \stackrel{\circ}{\mathrm{s}}(T-R-v)$. Since $\stackrel{\circ}{\mathrm{s}}\left(K_{1, r}\right)=r+u_{r}+1$ and $u_{r}=u_{r-p}$, the cost is at most $\stackrel{\circ}{\mathrm{S}}(T-R-v)+\stackrel{\circ}{\mathrm{s}}\left(K_{1, r}\right)$, which again is at most $\stackrel{\circ}{\mathrm{S}}(T-R)+r+u_{r}$.

Case 2: $v \in M$ and $p>u_{r}$. Let $M^{\prime}=M-(R \cup\{v\})$, let $X^{\prime}$ be an optimal response when Lister marks $M^{\prime}$ in $T^{\prime}$, and let $X=X^{\prime} \cup(M \cap R)$. Painter colors $X$. The final cost is at most $|M|+\stackrel{\circ}{\mathrm{s}}(T-X)$. We claim

$$
\stackrel{\circ}{\mathrm{s}}(T-X) \leq \stackrel{\circ}{\mathrm{s}}\left(T^{\prime}-X\right)+(r-p)+u_{r-p} .
$$

If $p<r$, then $v$ is a stem in $T-X$ and this follows from the induction hypothesis. If $p=r$, then $v$ has no leaf neighbors in $T-X$ and is not a stem, but then $T-X=T^{\prime}-X$ and $(r-p)+u_{r-p}=0$.

Also $|M|=\left|M^{\prime}\right|+p+1$, and $T^{\prime}-X=T^{\prime}-X^{\prime}$, and $u_{r-p} \leq u_{r}-1$ (since $p>u_{r}$ ), so

$$
\begin{aligned}
\stackrel{\circ}{\mathrm{S}}(T) \leq|M|+\stackrel{\circ}{\mathrm{S}}(T-X) & \leq\left|M^{\prime}\right|+p+1+\stackrel{\circ}{\mathrm{s}}\left(T^{\prime}-X\right)+(r-p)+u_{r-p} \\
& \leq\left|M^{\prime}\right|+\stackrel{\circ}{\mathrm{s}}\left(T^{\prime}-X^{\prime}\right)+r+u_{r} \leq \stackrel{\circ}{\mathrm{s}}\left(T^{\prime}\right)+r+u_{r} .
\end{aligned}
$$

Case 3: $v \in M$ and $p \leq u_{r}$. Let $M^{\prime}=M-R$, and let $X^{\prime}$ be an optimal response when Lister marks $M^{\prime}$ in $T^{\prime}$. (If $M^{\prime}=\varnothing$, then $X^{\prime}=\varnothing$.) If $v \in X^{\prime}$, then Painter colors $X^{\prime}$. Now $R$ consists of isolated vertices, so $\stackrel{\circ}{\mathrm{s}}(T) \leq|M|+\stackrel{\circ}{\mathrm{s}}\left(T^{\prime}-X^{\prime}\right)+r$. We compute

$$
|M|+\stackrel{\circ}{\mathrm{s}}\left(T^{\prime}-X^{\prime}\right)+r=p+\left|M^{\prime}\right|+\stackrel{\circ}{\mathrm{s}}\left(T^{\prime}-X^{\prime}\right)+r \leq \stackrel{\circ}{\mathrm{s}}\left(T^{\prime}\right)+p+r \leq \stackrel{\circ}{\mathrm{s}}\left(T^{\prime}\right)+r+u_{r} .
$$

If $v \notin X^{\prime}$, then Painter lets $X=X^{\prime} \cup(M \cap R)$ and colors $X$. The final score is at most $|M|+\stackrel{\circ}{\mathrm{s}}(T-X)$. Since $v$ remains a stem in $T-X$, the induction hypothesis yields $\stackrel{\circ}{\mathrm{s}}(T-X) \leq \stackrel{\circ}{\mathrm{s}}\left(T^{\prime}-X\right)+(r-p)+u_{r-p}$. Again $T^{\prime}-X=T^{\prime}-X^{\prime}$, so

$$
\begin{aligned}
|M|+\stackrel{\circ}{\mathrm{s}}(T-X) & \leq p+\left|M^{\prime}\right|+\stackrel{\circ}{\mathrm{s}}\left(T^{\prime}-X^{\prime}\right)+(r-p)+u_{r-p} \\
& \leq \stackrel{\circ}{\mathrm{s}}\left(T^{\prime}\right)+r+u_{r-p} \leq \stackrel{\circ}{\mathrm{s}}\left(T^{\prime}\right)+r+u_{r} .
\end{aligned}
$$

It remains only to prove equality in Lemma 3.2 when $r+1$ is triangular.

Lemma 3.3. If $r+1$ is triangular, then $\stackrel{\mathrm{s}}{\mathrm{s}}(T) \geq \stackrel{\circ}{\mathrm{s}}(T-R)+r+u_{r}$.

Proof. Again we use induction on $|V(T)|$. Again the claim holds when $v$ is the center of a star component, so we may assume that $v$ has a non-leaf neighbor. We give a strategy for Lister on the first move and obtain the desired lower bound for any Painter response.

Since $r+1$ is triangular and $r>0$, we have $r \geq u_{r}+1$. Let Lister's initial marked set $M$ consist of $v$ and $u_{r}+1$ vertices from $R$. By Observation 2.2, Painter responds by coloring $v$ or $M \cap R$. Lister plays optimally on the remaining graph. 
Case 1: Painter colors $v$. Let $T^{\prime}=T-R$; the final score is at least $|M|+r+\stackrel{\circ}{\mathrm{S}}\left(T^{\prime}-v\right)$. Since $v$ is a leaf in $T^{\prime}$, Lemma 2.3 yields $\stackrel{\circ}{\mathrm{s}}\left(T^{\prime}\right) \leq \stackrel{\circ}{\mathrm{s}}\left(T^{\prime}-v\right)+2$. Also $|M|=u_{r}+2$, so

$$
\stackrel{\circ}{\mathrm{s}}(T) \geq|M|+r+\stackrel{\circ}{\mathrm{s}}\left(T^{\prime}-v\right)=u_{r}+2+r+\stackrel{\circ}{\mathrm{s}}\left(T^{\prime}-v\right) \geq u_{r}+r+\stackrel{\circ}{\mathrm{s}}\left(T^{\prime}\right),
$$

Case 2: Painter colors $M \cap R$. Let $T^{\prime}=T-(M \cap R)$ and $r^{\prime}=r-\left(u_{r}+1\right)$. Since $r+1$ is triangular, also $r^{\prime}+1$ is triangular, and $u_{r^{\prime}}=u_{r}-1$.

First suppose $r>2$, so $r^{\prime}>0$. Now $v$ is a stem in $T^{\prime}$, with $r^{\prime}$ leaf vertices, and $T^{\prime}-R=T-R$. The final score is at least $|M|+\stackrel{\mathrm{s}}{(}\left(T^{\prime}\right)$. By the induction hypothesis,

$$
\begin{aligned}
\stackrel{\circ}{\mathrm{S}}(T) & \geq|M|+\stackrel{\circ}{\mathrm{S}}\left(T^{\prime}\right)=\left(u_{r}+2\right)+\left(u_{r^{\prime}}+r^{\prime}\right)+\stackrel{\circ}{\mathrm{S}}\left(T^{\prime}-R\right) \\
& =\left(u_{r}+2\right)+\left(u_{r}-1\right)+\left(r-u_{r}-1\right)+\stackrel{\circ}{\mathrm{s}}\left(T^{\prime}-R\right)=r+u_{r}+\stackrel{\circ}{\mathrm{s}}(T-R) .
\end{aligned}
$$

When $r=2$, we have $u_{r}=1$ and $M=R \cup\{v\}$. Thus $T^{\prime}=T-R$. We compute

$$
\stackrel{\circ}{\mathrm{S}}(T) \geq|M|+\stackrel{\circ}{\mathrm{s}}\left(T^{\prime}\right)=3+\stackrel{\circ}{\mathrm{s}}\left(T^{\prime}\right)=r+u_{r}+\stackrel{\circ}{\mathrm{s}}(T-R) .
$$

Together, Lemmas 3.13 .3 complete the proof of Theorem 1.2.

\section{Extremal Forests}

Theorem 1.2 makes it easy to characterize the $n$-vertex trees whose sum-color cost equals the upper bound $\lfloor 3 n / 2\rfloor$ proved in [10]. The characterization includes an alternative proof of that bound. We will do the same for the trees achieving the lower bound $n+u_{n-1}$.

The characterization of the upper bound when $n$ is even is simple and elegant, but for odd $n$ some annoying flexibility creeps in.

Theorem 4.1. If $T$ is an $n$-vertex forest, then $\stackrel{\mathrm{S}}{(} T) \leq\lfloor 3 n / 2\rfloor$. Furthermore, equality holds if and only if $T$ contains a spanning forest in which every vertex has degree 1 or 3 , except that when $n$ is odd there is also one vertex with degree 0 or 6.

Proof. Say that a forest is tight if it contains a spanning forest as described in the statement. Call such a spanning forest a witness. Note that a witness has a vertex of even degree if and only if $n$ is odd. Say that a tree is even when the number of vertices is even; otherwise it is odd.

We use induction on $n$. The claims hold by inspection when $T$ has no edges, including when $T$ has no vertices. Hence we may assume that $T$ has a stem $v$ (possibly the center of a star component). Let $R$ be the set of leaf neighbors of $v$, with $r=|R|$, and let $T^{\prime}=T-R-v$.

We first prove the upper bound, using Theorem 1.2. Note that $r+1+u_{r} \leq\lfloor 3(r+1) / 2\rfloor$, with equality only for $r \in\{1,2,3,4,6\}$. If $r+1$ is not triangular, then the induction hypothesis yields $\stackrel{\circ}{\mathrm{s}}(T)=\stackrel{\circ}{\mathrm{s}}\left(T^{\prime}\right)+r+1+u_{r} \leq\lfloor 3(n-r-1) / 2\rfloor+\lfloor 3(r+1) / 2\rfloor \leq\lfloor 3 n / 2\rfloor$. If $r+1$ is triangular, then $r+u_{r} \leq\lfloor 3 r / 2\rfloor$, with equality (among triangular $r+1$ ) only for $r \in\{2,5\}$. Hence when $r+1$ is triangular, the induction hypothesis yields $\stackrel{\mathrm{s}}{\mathrm{S}}(T)=$ $\stackrel{\circ}{\mathrm{s}}(T-R)+r+u_{r} \leq\lfloor 3(n-r) / 2\rfloor+\lfloor 3 r / 2\rfloor \leq\lfloor 3 n / 2\rfloor$. 
This completes the proof of the upper bound. It remains to prove the characterization of equality (again by induction, with the basis when $T$ has no edges). Some cases in the proof are shown in Figure1. The upper bound implies that when proving sufficiency, we only need to prove $\stackrel{\circ}{s}(T) \geq\lfloor 3 n / 2\rfloor$ when $T$ is tight.

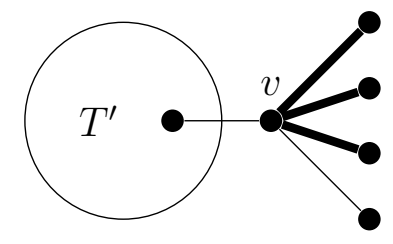

(a)

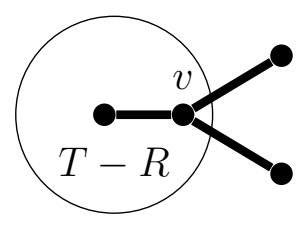

(b)

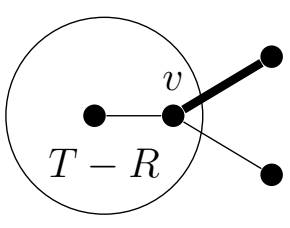

(c)

Figure 1: Selected cases for Theorem 4.1.

Necessity. We assume $\stackrel{\circ}{\mathrm{s}}(T)=\lfloor 3 n / 2\rfloor$. The upper bound computation yields $r \leq 6$.

If $r+1$ is not triangular, then $r \in\{1,3,4,6\}$. Equality in the bound requires $\stackrel{\circ}{\mathrm{s}}\left(T^{\prime}\right)=$ $\left\lfloor 3\left|V\left(T^{\prime}\right)\right| / 2\right\rfloor$, so by the induction hypothesis $T^{\prime}$ is tight. Thus $T^{\prime}$ has a witness. If $r \in\{1,3\}$, then adding the edges from $v$ to $R$ yields a witness for $T$. If $r \in\{4,6\}$, then $\lfloor 3(r+1) / 2\rfloor<$ $3(r+1) / 2$, so equality in $\stackrel{\circ}{\mathrm{s}}(T) \leq\lfloor 3 n / 2\rfloor$ requires $T^{\prime}$ to be even. Hence a witness for $T^{\prime}$ has no vertex of even degree, and we obtain a witness for $T$ by adding three edges from $v$ to $R$ if $r=4$ and adding all six edges if $r=6$. Figure 1(a) shows the case $r=4$.

Now suppose $r+1$ is triangular, so $r \in\{2,5\}$. Equality requires $\stackrel{\circ}{(}(T-R)=\left\lfloor 3\left|V\left(T^{\prime}\right)\right| / 2\right\rfloor$, and hence $T-R$ is tight. When $r=5$, we have $\lfloor 3 r / 2\rfloor=(3 r-1) / 2$, so $\stackrel{\mathrm{s}}{\mathrm{s}}(T)=\lfloor 3 n / 2\rfloor$ requires $T-R$ to be even. Hence $v$ has degree 1 in a witness for $T-R$, and adding all the edges from $v$ to $R$ completes a witness for $T$, with $v$ of degree 6 .

When $r=2$, again $T-R$ must be tight. If a witness has degree 1 at $v$, as in Figure 1(b), then add both edges from $v$ to $R$ to make a witness for $T$. If instead $v$ is isolated, as in Figure 1(c), then add one edge from $v$ to $R$ and leave the other vertex of $R$ isolated.

Sufficiency. We assume that $T$ is tight.

If a witness $W$ for $T$ has some vertex $z \in R$ as an isolated vertex, then $W-z$ is a witness for $T-z$, and $T-z$ is even. By the induction hypothesis, $\stackrel{\mathrm{s}}{(}(T-z)=3(n-1) / 2$. Since Lister can play $T-z$ and $z$ separately, $\stackrel{\circ}{\mathrm{s}}(T) \geq\lfloor 3 n / 2\rfloor$, and hence equality holds.

Hence we may assume that all vertices of $R$ have degree 1 in $W$. This requires $d_{W}(v) \in$ $\{1,3,6\}$. If $d_{W}(v)=r$, then $r+1$ is not triangular. Also $T^{\prime}$ is tight (and is even if $d_{W}(v)=6$ ). By the induction hypothesis, equality holds for $T^{\prime}$ and in the computation for $T$.

Hence we may assume that $v$ has a non-leaf neighbor in $W$, so $r=d_{W}(v)-1$, which eliminates the case $r=1$. Note that $r+1 \in\{3,6\}$, so $r+1$ is triangular, and $\stackrel{\mathrm{s}}{\mathrm{s}}(T)=$

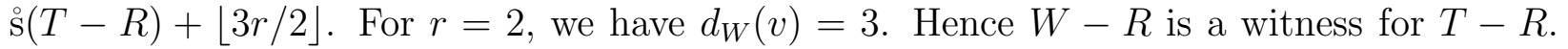
When $r=5$ and $d_{W}(v)=6$, tightness of $T$ requires $T$ to be odd. Deleting the five vertices of $R$ from $W$ leaves a witness for $T-R$. In both cases, the induction hypothesis gives equality hold in the bound for $T-R$ and hence in the bound for $T$. 
Our final task is to characterize the $n$-vertex trees having the least cost. When neither $n-1$ nor $n-2$ is triangular, the star $K_{1, n-1}$ is the unique minimizing tree, but in the remaining cases a few other trees may also have this cost. For example, from Lemma 3.1 and the formula $\stackrel{\circ}{\mathrm{s}}\left(K_{1, r}\right)=r+1+u_{r}$, it is easy to check that when $n-1$ or $n-2$ is triangular the tree obtained by subdividing one edge of $K_{1, n-2}$ has the same cost as $K_{1, n-1}$.

Our proof of the characterization provides an alternative proof of the result in [10] that the minimum is $n+u_{n-1}$. We begin with a numerical lemma, which suggests that the tree obtained by subdividing one edge of $K_{1, n-2}$ is the best candidate to match $K_{1, n-1}$.

Lemma 4.2. If $m, r \in \mathbb{N}$ satisfy $2<r \leq m / 2$, then $u_{r}+u_{m-r} \geq u_{1}+u_{m-1}$. If also no number in $\{m-4, m-3, m-2, m-1\}$ is triangular, then $u_{r}+u_{m-r} \geq 1+u_{1}+u_{m-1}$.

Proof. Let $a_{r}=u_{r}+u_{m-r}$. To prove the first statement, it suffices to show for $2<r \leq m / 2$ that there exists $q$ with $1 \leq q<r$ and $q \neq 2$ such that $a_{q} \leq a_{r}$.

First consider $r \in\{3,4,5\}$ and $m \geq 2 r$. We have $a_{1}=1+u_{m-1}$ and $a_{r}=2+u_{m-r}$. When $r \in\{3,4,5\}$ and $m \geq 2 r$, there cannot be two triangular numbers in the interval $[m-r+1, m-1]$, so $u_{m-r} \geq u_{m-1}-1$. Thus $a_{1} \leq a_{r}$, so setting $q=1$ suffices.

For $r \geq 6$, first compare $a_{r}$ with $a_{r-1}$ :

$$
a_{r}-a_{r-1}= \begin{cases}1 & \text { if } r \text { is triangular and } m-r+1 \text { is not } \\ -1 & \text { if } m-r+1 \text { is triangular and } r \text { is not } \\ 0 & \text { otherwise. }\end{cases}
$$

If $m-r+1$ is not triangular or $r$ is triangular, then setting $q=r-1$ suffices. Otherwise, $m-r+1$ is triangular and $r$ is not. Let $q=\left(\begin{array}{c}u_{r}+1 \\ 2\end{array}\right)-1$; note that $u_{q}=u_{r}-1$. Also, the difference between $m-r+1$ and the next higher triangular number is greater than $r-q$; hence $u_{m-q}=u_{m-r+1}=u_{m-r}+1$. Thus $a_{q}=a_{r}$. Also, since $r>6$, we have $q \geq 5$, so we eventually reduce to $r=5$. This proves the first statement.

Now suppose that none of $\{m-4, m-3, m-2, m-1\}$ is triangular. In this case, for $r \in\{3,4,5\}$, there is no triangular number in the interval $[m-r+1, m-1]$; hence $u_{m-r}=u_{m-1}$ and $a_{r} \geq a_{1}+1$. For $r \geq 6$, the argument above yields $q$ with $r>q \geq 5$ such that $a_{q} \leq a_{r}$, which now implies the stronger inequality $a_{r} \geq a_{1}+1$.

Theorem 4.3. If $T$ is an $n$-vertex tree, then $\stackrel{\mathrm{S}}{(} T) \geq n+u_{n-1}$, with equality for $T=K_{1, n-1}$. Furthermore, $K_{1, n-1}$ is the unique minimizing tree when neither $n-1$ nor $n-2$ is triangular.

Proof. We proved $\stackrel{\circ}{\mathrm{S}}\left(K_{1, n-1}\right)=n+u_{n-1}$ in Theorem 2.5. To complete the proof, we show that $\stackrel{\circ}{\mathrm{S}}(T) \geq \stackrel{\circ}{\mathrm{S}}\left(K_{1, n-1}\right)$ for every non-star tree, with strict inequality when neither $n-1$ nor $n-2$ is triangular. We use induction on $n$. Every tree with at most three vertices is a star, so we may assume $n \geq 4$.

Let $v$ be a stem in $T$, and let $R$ be the set of leaf neighbors of $v$, with $r=|R|$. Since we may let $v$ be the penultimate vertex on either end of a longest path, we may assume $r \leq(n-2) / 2$. Let $m=n-2$. 
We claim $\stackrel{\circ}{s}(T) \geq n+1+u_{n-3}$. If so, then since $n \geq 4$ implies $u_{n-3} \geq u_{n-1}-1$, we have $\stackrel{\circ}{\mathrm{s}}(T) \geq \stackrel{\circ}{\mathrm{s}}\left(K_{1, n-1}\right)$. If also neither $n-1$ nor $n-2$ is triangular, then $u_{n-3}=u_{n-1}$, and $\stackrel{\mathrm{S}}{ }(T)>\stackrel{\circ}{\mathrm{s}}\left(K_{1, n-1}\right)$. It remains to prove the claim.

If $r=2$, then since $r+1$ is triangular, Theorem 1.2 and the induction hypothesis yield

$$
\stackrel{\circ}{\mathrm{s}}(T)=\stackrel{\circ}{\mathrm{s}}(T-R)+r+u_{r}=\stackrel{\circ}{\mathrm{s}}(T-R)+3 \geq\left(n-2+u_{n-3}\right)+3=n+u_{n-3}+1 .
$$

If $r \neq 2$, then we use Theorem 1.2, the lower bound in Lemma 2.3 (yielding $\stackrel{\mathrm{s}}{ }(T-R) \geq$ $\stackrel{\mathrm{s}}{ }(T-R-v)+1)$, the induction hypothesis, and Lemma 4.2 for $r \neq 2$ (with $m=n-2$ ) to compute

$$
\begin{aligned}
\stackrel{\circ}{\mathrm{S}}(T) \geq \stackrel{\circ}{\mathrm{s}}(T-R-v)+r+1+u_{r} & \geq n-r-1+u_{n-r-2}+r+1+u_{r} \\
& =n+u_{r}+u_{n-r-2} \geq n+1+u_{n-3} .
\end{aligned}
$$

This completes the proof.

The remaining case is somewhat technical; additional minimizing trees arise. They are close to being stars. For $a, b \geq 1$, the double-star $S_{a, b}$ is the tree with $a+b+2$ vertices having two non-leaf vertices, one with $a$ leaf neighbors and the other with $b$ leaf neighbors (see Figure 21). The subdivided double-star $S_{a, b}^{\prime}$ is obtained from $S_{a, b}$ by subdividing the central edge to add one vertex. Note that the tree obtained from $K_{1, n-2}$ by sudividing one edge is $S_{1, n-3}$.

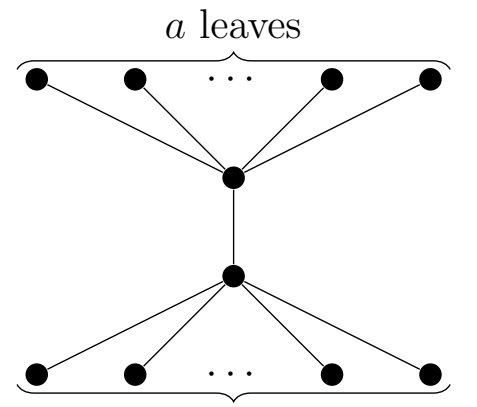

$b$ leaves

$S_{a, b}$

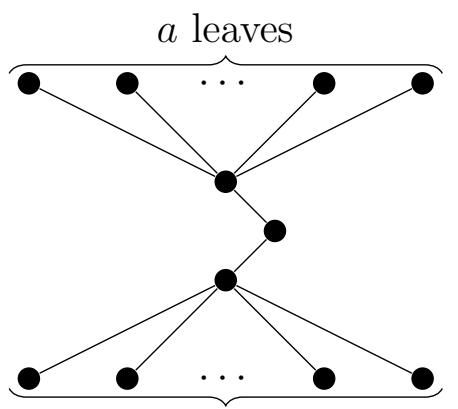

$b$ leaves

Figure 2: Non-star trees achieving the lower bound in Theorem 4.4.

Theorem 4.4. When $n-1$ or $n-2$ is triangular, the $n$-vertex trees $T$ minimizing $\stackrel{\circ}{\mathrm{s}}(T)$ are $\left\{K_{1, n-1}, S_{1, n-3}, S_{2, n-4}, S_{1, n-4}^{\prime}\right\}$, except that for $n=7$ all trees are included, and $S_{4,5}$ and $S_{4,4}^{\prime}$ are included when $n=11$.

Proof. For $n \in\{4,5,7\}$, the upper and lower bounds on $\stackrel{\circ}{\mathrm{s}}(T)$ are equal, so all $n$-vertex trees achieve the lower bound. When $n \in\{4,5\}$, all $n$-vertex trees are in the listed set. Thus, we may use induction on $n$ with basis $n \leq 7$. Suppose $n \geq 8$. 
Let $T$ be an $n$-vertex tree achieving $\stackrel{\circ}{\mathrm{S}}(T)=n+u_{n-1}$. Let $v$ be a stem having the fewest leaf neighbors, $R$ the set of leaves adjacent to $v$, and $r=|R|$. We may assume that $T$ is not a star, which implies $r \leq \frac{n-2}{2}$.

Since $n-1$ or $n-2$ is triangular, $u_{n-3}=u_{n-1}-1$. Furthermore, since $n \geq 8$, the numbers $n-3$ and $n-4$ are not triangular, which means $u_{n-4}=u_{n-5}=u_{n-1}-1$.

If $r=1$, then $r+1$ is not triangular, and Theorem 1.2 yields $\stackrel{\mathrm{s}}{(}(T)=\stackrel{\mathrm{s}}{ }(T-R-v)+3$. Also $T-R-v$ has $n-2$ vertices, so $\stackrel{\circ}{\mathrm{s}}(T) \geq(n-2)+u_{n-3}+3=n+u_{n-1}$. Since neither $n-3$ nor $n-4$ is triangular, by the induction hypothesis equality holds if and only if $T-R-v=K_{1, n-3}$. Depending on whether $v$ is adjacent to the center or a leaf of $T-R-v$, we have equality if and only if $T$ is $S_{1, n-3}$ or $S_{1, n-4}^{\prime}$.

If $r=2$, then $r+1$ is triangular, and Theorem 1.2 yields $\stackrel{\circ}{\mathrm{s}}(T)=\stackrel{\circ}{\mathrm{s}}(T-R)+3$. Now $T-R$ has $n-2$ vertices, so the same argument as above yields $T-R=K_{1, n-3}$ when equality holds. Our vertex $v$ lies in $K_{1, n-3}$, and $T$ is obtained by adding two pendant edges at $v$. If $v$ is the center of $K_{1, n-3}$, then $T$ is a star; hence $v$ is a leaf of $K_{1, n-3}$ and $T=S_{2, n-4}$.

Hence we may assume $r \geq 3$. Let $\epsilon=1$ if $\{n-3, n-4, n-5, n-6\}$ contains no triangular number, and otherwise $\epsilon=0$. As in Theorem 4.3, we use the lower bound in Lemma 2.3, the lower bound for trees with $n-r-1$ vertices, Lemma 4.2 (with $m=n-2$ ), and $u_{n-3}=u_{n-1}-1$ to compute

$$
\stackrel{\circ}{\mathrm{S}}(T) \geq \stackrel{\circ}{\mathrm{S}}(T-R-v)+r+1+u_{r}=n+u_{n-r-2}+u_{r} \geq n+\epsilon+u_{n-3}+u_{1}=n+\epsilon+u_{n-1} .
$$

If $\epsilon=1$, then $\stackrel{\mathrm{s}}{(} T)>\stackrel{\circ}{\mathrm{s}}\left(K_{1, n-1}\right)$. Since $n-1$ or $n-2$ is triangular, when $n \geq 17$ no triangular number lies in $\{n-3, n-4, n-5, n-6\}$. The only cases remaining are $n \in\{8,11,12,16\}$, which yield $\epsilon=0$. Now equality must hold throughout in (1), which requires $\stackrel{\circ}{\mathrm{S}}(T-R-v)=$ $(n-r-1)+u_{n-r-2}$. In other words, $T-R-v$ is a minimizing tree on $n-r-1$ vertices, and $\stackrel{\circ}{\mathrm{s}}(T)=n+u_{n-r-2}+u_{r}$. Equaling the minimum requires $u_{n-r-2}+u_{r}=u_{n-1}$.

If $n=8$, then $r \leq \frac{n-2}{2}$ forces $r=3$. (Recall that we are assuming $r \geq 3$ here.) Since $u_{3}+u_{3}=4>3=u_{7}$, the only minimizing trees are $\left\{K_{1,7}, S_{1,5}, S_{2,4}, S_{1,4}^{\prime}\right\}$.

If $n=11$, then $r \leq \frac{n-2}{2}$ forces $r \leq 4$. Since $u_{6}+u_{3}=5>4=u_{10}$, the case $r=3$ is eliminated. If $r=4$, then $\stackrel{\mathrm{s}}{(} T)=11+u_{5}+u_{4}=15$, and $T-R-v$ is a minimizing tree on six vertices. Thus $T-R-v=K_{1,5}$, which yields $T \in\left\{S_{4,5}, S_{4,4}^{\prime}\right\}$, depending on whether $v$ is adjacent to the center or a leaf of $K_{1,5}$.

If $n=12$, then $r \leq \frac{n-2}{2}$ forces $r \leq 5$. Note that $u_{7}+u_{3}=u_{6}+u_{4}=5>4=u_{11}$, so we may assume $r=5$. Since $r+1$ is triangular, Theorem 1.2 eliminates this case via

$$
\stackrel{\circ}{\mathrm{S}}(T)=\stackrel{\circ}{\mathrm{s}}(T-R)+5+u_{5} \geq 7+u_{6}+5+u_{5}=17 .
$$

If $n=16$, then $r \leq \frac{n-2}{2}$ forces $r \leq 7$. Since $u_{r}+u_{14-r}=6>5=u_{15}$ for $r \in\{3,4,6,7\}$, such values of $r$ cannot occur in a minimizing tree. When $r=5$ we again apply Theorem 1.2 to eliminate this case via

$$
\stackrel{\circ}{\mathrm{S}}(T)=\stackrel{\circ}{\mathrm{s}}(T-R)+5+u_{5} \geq 11+u_{10}+5+u_{5}=22>21=16+u_{15} .
$$

This completes the proof.

Theorems 4.3 and 4.4 complete the characterization of the minimizing trees. 


\section{The Interactive Sum Choice Number of Forests}

Recall that in the game introduced by Bonamy and Meeks [2], in each round Requester specifies a vertex $v$ of the graph $G$ and Supplier adds a color to the list $L(v)$, with the game ending when $G$ is $L$-colorable. The length of the game under optimal play is $\chi_{\mathrm{ISC}}(G)$.

We prove $\stackrel{\mathrm{S}}{\mathrm{S}}(T)=\chi_{\mathrm{ISC}}(T)$ for each forest $T$ by showing that $\chi_{\mathrm{ISC}}$ satisfies the same recurrence as $\stackrel{\circ}{\mathrm{s}}$ on trees, using lemmas like those in Section 3 . That is, we prove the following:

Theorem 5.1. Let $T$ be a forest. If $T$ has no edges, then $\chi_{\operatorname{ISC}}(T)=|V(T)|$. If $v$ is a stem in $T$ and $R$ is the set of leaf neighbors of $v$, with $r=|R|$, then

$$
\chi_{\mathrm{ISC}}(T)= \begin{cases}\chi_{\mathrm{ISC}}(T-R-v)+r+1+u_{r}, & \text { if } r+1 \text { is not a triangular number }, \\ \chi_{\mathrm{ISC}}(T-R)+r+u_{r}, & \text { if } r+1 \text { is a triangular number. }\end{cases}
$$

Interactive sum coloring satisfies bounds like Lemma 2.3 for slow coloring when a graph is broken into subgraphs by a vertex bipartition. The lemma is a special case of one by Bonamy and Meeks [2], which we rephrase slightly. We include a proof for completeness.

Lemma 5.2 (Bonamy-Meeks [2]). If $G$ is a graph and $(A, B)$ is a partition of $V(G)$, then

$$
\chi_{\mathrm{ISC}}(G[A])+\chi_{\mathrm{ISC}}(G[B]) \leq \chi_{\mathrm{ISC}}(G) \leq \chi_{\mathrm{ISC}}(G[A])+\chi_{\mathrm{ISC}}(G[B])+|[A, B]| .
$$

Proof. As in Lemma 2.3, the lower bound holds because Supplier can respond in the games on $G[A]$ and $G[B]$ separately. For the upper bound, Requester can play optimal strategies first on $G[A]$ (producing a proper coloring $\phi$ from the resulting lists) and then on $G[B]$, with an extra request made at the endpoint $y$ in $B$ of an edge $x y$ in $[A, B]$ whenever Supplier provides the color $\phi(x)$ at $y$. Supplier can only provide that color once for each such edge, and Requester can put aside that response and continue on $G[B]$ as if it never happened.

Recall from Theorem 1.4 that $\chi_{\mathrm{ISC}}\left(K_{1, r}\right)=r+1+u_{r}$. Also $\chi_{\mathrm{ISC}}(G)=|V(G)|$ when $|V(G)| \leq 1$. When $T$ is a star, both cases in Theorem 5.1 give the known formula $\chi_{\mathrm{ISC}}(T)=$ $r+1+u_{r}$. Also the parameter is additive over components. Thus, we may assume that $T$ has a component that is not a star and thus has a stem with a non-leaf neighbor.

Henceforth $v$ is a stem with non-leaf neighbor $w$ in a non-star component of a forest $T$, the set of leaf neighbors of $v$ is $R$, and $r=|R| \geq 1$. Let $R^{\prime}=R \cup\{v\}$ and $T^{\prime}=T-R^{\prime}=$ $T-R-\{v\}$. Lemma 5.2 and Theorem 1.4 yield

$$
\chi_{\mathrm{ISC}}\left(T^{\prime}\right)+r+u_{r}+1 \leq \chi_{\mathrm{ISC}}(T) \leq \chi_{\mathrm{ISC}}\left(T^{\prime}\right)+r+u_{r}+2 .
$$

For Theorem 5.1, in the case where $r+1$ is not triangular we need a strategy for Requester that improves the upper bound in (2) by 1 .

Definition 5.3. Given a stem $v$ with a color $c$ in its current list, freeing $c$ at $v$ means requesting an additional color at each leaf neighbor of $v$ whose current list is precisely $\{c\}$.

Note that freeing $c$ at $v$ may make $c$ available for use at $v$ in a proper coloring chosen from the lists; it also ensures that each leaf neighbor of $v$ with $c$ in its list can be colored. 
Lemma 5.4. If $r+1$ is not triangular, then $\chi_{\mathrm{ISC}}(T)=\chi_{\mathrm{ISC}}\left(T^{\prime}\right)+r+u_{r}+1$.

Proof. Due to (2), it suffices to prove $\chi_{\mathrm{ISC}}(T) \leq \chi_{\mathrm{ISC}}\left(T^{\prime}\right)+r+u_{r}+1$. We provide a strategy for Requester that is a slight modification of the strategy used on stars in [2].

Requester first requests an initial color at each vertex of $R^{\prime}$. For each $x \in R^{\prime}$, let $\alpha(x)$ be the first color supplied by Supplier at $x$. Requester's subsequent strategy is in three phases. Phases 1 and 3 involve making requests in $R^{\prime}$; Phase 2 involves playing optimally on $T^{\prime}$. During the game, let $i$ denote the number of requests that have been made so far at $v$, let $c_{i}$ be the color supplied in response to the $i$ th request at $v$, and let $S_{i}=\left\{z \in R: \alpha(z)=c_{i}\right\}$. After the initial colors are supplied at $R^{\prime}$, we have $i=1$ and $c_{1}=\alpha(v)$.

Phase 1. Phase 1 requests colors at $v$. As long as $\left|S_{i}\right|>u_{r}-i+1$, Requester obtains color $c_{i+1}$ at $v$ and increments $i$. When $\left|S_{i}\right| \leq u_{r}-i+1$, no request is made, Phase 2 begins, and we set $i^{*}=i$. There is no request in Phase 1 if $\left|S_{1}\right| \leq u_{r}$.

Phase 2. Requester plays on $T^{\prime}$. There are two cases, depending on whether $\left|S_{i}\right|$ equals $u_{r}-i+1$ or is smaller.

If $\left|S_{i^{*}}\right| \leq u_{r}-i^{*}$, then Requester first frees $c_{i^{*}}$ at $v$ and then plays an optimal request sequence on $T^{\prime}$. However, if Supplier adds $c_{i^{*}}$ to the list at $w$ in response to a request there, then Requester ignores that move and immediately makes an extra request at $w$. The copy of $c_{i^{*}}$ becomes an extra unused color in $L(w)$. In the game played optimally by Requester on $T^{\prime}$, the list at $w$ is considered not to contain $c_{i^{*}}$. An $L$-coloring $\phi$ of $T^{\prime}$ then exists without using $c_{i^{*}}$ at $w$. With $c_{i^{*}}$ available at $v$, the proper coloring $\phi$ extends to $R^{\prime}$.

If $\left|S_{i^{*}}\right|=u_{r}-i^{*}+1$, then Requester next plays an optimal request sequence on $T^{\prime}$. From the colors supplied, Requester can choose a proper coloring $\phi$ of $T^{\prime}$. Let $c^{\prime}=\phi(w)$. If $c^{\prime} \neq c_{i^{*}}$, then Requester frees $c_{i^{*}}$ at $v$ (by adding colors at the leaf neighbors of $v$ in $R$ ), which makes it possible to use $c_{i^{*}}$ at $v$ and extend $\phi$ to all of $T$. If $c^{\prime}=c_{i^{*}}$, then the process moves to Phase 3.

Phase 3. Here, in the situation $\left|S_{i^{*}}\right|=u_{r}-i^{*}+1$ and $c^{\prime}=c_{i^{*}}$, Requester increments $i$ (to $i^{*}+1$ ) and requests $c_{i^{*}+1}$ at $v$. As long as $\left|S_{i}\right|>u_{r}-i+1$, Requester increments $i$ and requests a color at $v$. At the point when $\left|S_{i}\right| \leq u_{r}-i+1$, Requester frees $c_{i}$ at $v$. Since $c^{\prime}=\phi(w)=c_{i^{*}}$, the new color at $v$ is different from the color $c^{\prime}$ at $w$ under $\phi$, so it can be used at $v$ and the coloring extends to all of $T$.

Any time a request is made in Phase 1 or Phase 3 to pick another color at $v$ after $c_{i}$, we have $\left|S_{i}\right| \geq u_{r}-i+2$. If we reach Phase 3 , then we also have $\left|S_{i^{*}}\right|=u_{r}-i^{*}+1$. The sets of the form $S_{i}$ are pairwise disjoint subsets of $R$. There are at most $u_{r}$ requests in Phases 1 and 3, since making $u_{r}+1$ such requests requires $r=|R| \geq \sum_{i=1}^{u_{r}+1}\left|S_{i}\right| \geq\left(\begin{array}{c}u_{r}+2 \\ 2\end{array}\right)-1$. Since $r+1$ is not triangular, we also have $r \geq\left(\begin{array}{c}u_{r}+2 \\ 2\end{array}\right)$, but by definition $u_{r}=\max \left\{k:\left(\begin{array}{c}k+1 \\ 2\end{array}\right) \leq r\right\}$. Hence the strategy terminates, with at most $1+u_{r}$ requests at $v$.

We have observed that this strategy produces lists from which a proper coloring can be chosen on $T$, given an optimal strategy on $T^{\prime}$. We claim also that it uses at most $r+u_{r}+1+\chi_{\mathrm{ISC}}\left(T^{\prime}\right)$ requests altogether.

Suppose first that $\left|S_{i^{*}}\right| \leq u_{r}-i^{*}$. In this case, we claim that the total number of requests is at most $r+i^{*}+\left|S_{i^{*}}\right|+\left(\chi_{\mathrm{ISC}}\left(T^{\prime}\right)+1\right)$. After the initial $r+1$ requests on $R^{\prime}$ come $i^{*}-1$ 
additional requests at $v$ in Phase 1 . Freeing $c_{i^{*}}$ at $v$ in Phase 2 does not make a request at $v$ but makes $\left|S_{i^{*}}\right|$ requests in $R$. There are then at most $\chi_{\mathrm{ISC}}\left(T^{\prime}\right)+1$ requests on $T^{\prime}$ in Phase 2 (there may be an extra request at $w$ ). Since $\left|S_{i^{*}}\right| \leq u_{r}-i^{*}$, the total number of requests is at most $r+u_{r}+1+\chi_{\mathrm{ISC}}\left(T^{\prime}\right)$, as desired.

If $\left|S_{i^{*}}\right|=u_{r}-i^{*}+1$, then let $c_{k}$ be the last color supplied at $v$. We claim that the total number of requests is at most $r+k+\left|S_{k}\right|+\chi_{\mathrm{ISC}}\left(T^{\prime}\right)$. Initially there are $r+1$ requests on $R^{\prime}$, and eventually there are $k-1$ additional requests at $v$. In Phase 2 there are at most $\chi_{\mathrm{ISC}}\left(T^{\prime}\right)$ requests on $T^{\prime}$. If $c^{\prime} \neq c_{i^{*}}$, then $i^{*}=k$, so freeing $c_{i^{*}}$ uses $\left|S_{k}\right|$ additional requests on $R$. On the other hand, if $c^{\prime}=c_{i^{*}}$, then later freeing $c_{k}$ at $v$ incurs $\left|S_{k}\right|$ additional requests on $R$; again the claimed bound holds. In both cases $\left|S_{k}\right| \leq u_{r}-k+1$, yielding at most $r+1+u_{r}+\chi_{\mathrm{ISC}}\left(T^{\prime}\right)$ total requests.

When $r+1$ is triangular, we want to compute $\chi_{\mathrm{ISC}}(T)$ in terms of the subtree obtained by deleting only $R$, not $R \cup\{v\}$. That is, $\chi_{\text {ISC }}(T)=\chi_{\text {ISC }}(T-R)+r+u_{r}$. The upper bound actually does not depend on $r+1$ being triangular.

Lemma 5.5. $\chi_{\mathrm{ISC}}(T) \leq \chi_{\mathrm{ISC}}(T-R)+r+u_{r}$.

Proof. We give a strategy for Requester. The strategy is similar to that in in Lemma 5.4, except that in Phase 2 we play a subgame on $T-R$ instead of $T-R-v$. Again Requester first requests an initial color $\alpha(x)$ for each $x \in R^{\prime}$. Again let $c_{i}$ be the $i$ th color supplied at $v$, and let $S_{i}=\left\{z \in R: \alpha(z)=c_{i}\right\}$.

Phase 1. As long as $\left|S_{i}\right|>u_{r}-i+1$, Requester obtains color $c_{i+1}$ at $v$ and increments $i$. When $\left|S_{i}\right| \leq u_{r}-i+1$, no request is made, Phase 2 begins, and we set $i^{*}=i$.

Phase 2. There are two cases, depending on whether $\left|S_{i^{*}}\right|$ equals $u_{r}-i^{*}+1$ or is smaller.

If $\left|S_{i^{*}}\right| \leq u_{r}-i^{*}$, then Requester proceeds as in Lemma 5.4. Requester frees $c_{i^{*}}$ at $v$ and then plays optimally on $T^{\prime}$. Again if Supplier adds $c_{i^{*}}$ to the list at $w$ in response to a request there, then Requester makes an extra request at $w$. Without using the copy of $c_{i^{*}}$ in $L(w)$, Requester obtains lists on $T^{\prime}$ from which a proper coloring can be chosen. With $c_{i^{*}}$ available at $v$, this coloring $\phi$ extends to $R^{\prime}$.

If $\left|S_{i^{*}}\right|=u_{r}-i^{*}+1$, then Requester next plays an optimal game on $T-R$, treating $L(v)$ as initially empty in the game on $T-R$. The first time Requester's optimal strategy on $T-R$ makes a request at $T-R$, we treat it in the game on $T-R$ as being supplied by the color $c_{i}^{*}$ at $v$ that was supplied earlier. Hence we save one from $\chi_{\mathrm{ISC}}(T-R)$ in counting the requests. From the resulting lists, choose a proper coloring $\phi$ of $T-R$, using $c_{i^{*}}$ at $v$ if possible. Since $v$ is a leaf in $T-R$, this is possible unless $c_{i^{*}}$ must be used at $w$. Let $c^{\prime}=\phi(v)$. If $c^{\prime}=c_{i^{*}}$, then Requester frees $c_{i^{*}}$ at $v$ and extends $\phi$ to all of $T$. If $c^{\prime} \neq c_{i^{*}}$, then the process moves to Phase 3.

Phase 3. Here, in the situation $\left|S_{i^{*}}\right|=u_{r}-i^{*}+1$ and $c^{\prime} \neq c_{i^{*}}$, Requester increments $i$ (to $i^{*}+1$ ) and requests $c_{i^{*}+1}$ at $v$. As long as $\left|S_{i}\right|>u_{r}-i+1$, Requester increments $i$ and requests a color at $v$. At the point when $\left|S_{i}\right| \leq u_{r}-i+1$, Requester frees $c_{i}$ at $v$. Since color $c_{i^{*}}$ already exists in $L(v)$ and has been used at $\phi(w)$, the color $c_{i}$ is different from $c_{i^{*}}$ and can be used at $v$. With $\phi(v)=c_{i}$, the coloring now extends to all of $T$. 
The same counting argument as in Lemma 5.4 shows that the strategy terminates, and we have argued that it produces lists from which a proper coloring can be chosen. We claim that it makes at most $\chi_{\mathrm{ISC}}(T-R)+r+u_{r}$ requests.

If $\left|S_{i^{*}}\right| \leq u_{r}-i^{*}$, then as argued in Lemma 5.4 the total number of requests is at most $r+i^{*}+\left|S_{i^{*}}\right|+\left(\chi_{\mathrm{ISC}}\left(T^{\prime}\right)+1\right)$. Since $\left|S_{i^{*}}\right| \leq u_{r}-i^{*}$, the number of requests is at most $r+u_{r}+1+\chi_{\mathrm{ISC}}\left(T^{\prime}\right)$. Since $\chi_{\mathrm{ISC}}(T-R) \geq \chi_{\mathrm{ISC}}\left(T^{\prime}\right)+1$ by (2), we obtain $\chi_{\mathrm{ISC}}(T) \leq$ $r+u_{r}+\chi_{\mathrm{ISC}}(T-R)$, as desired.

If $\left|S_{i^{*}}\right|=u_{r}-i^{*}+1$, then let $c_{k}$ be the last color supplied at $v$. Now the total number of requests is at most $r+k+\left|S_{k}\right|+\left(\chi_{\mathrm{ISC}}(T-R)-1\right)$, where as remarked earlier we save one request on $T-R$ because Requester uses the already-counted first request made at $v$. Since $\left|S_{k}\right| \leq u_{r}-k+1$, again the desired bound holds.

Finally, we prove the lower bound in the triangular case. To simplify arguments for optimal strategies for Supplier, we prove a lemma with two statements about a fixed vertex $v$ in the game on any graph $G$. The first property is that Supplier has an optimal strategy with the freedom to name the first color supplied at $v$ independently of what Requester does. This property is a special case of Observation 2.1 of Bonamy and Meeks [2]. We include a proof not only for completeness, but also to show that this and the second property can be guaranteed simultaneously.

The second property restricts the strategies that need to be considered for Requester in response, showing that it is non-optimal for Requester to make too many requests at one vertex. The goal of Requester on a graph $G$ is to produce a list assignment $L$ such that $G$ is $L$-colorable. Just as it is useless in $f$-choosability to have $f(v) \geq d(v)+1$, where $d(v)$ denotes the degree of $v$ in $G$, so it is nonoptimal for Requester to request more than $d(v)+1$ colors at a vertex. Such requests allow Supplier to increase the final score.

Note that once a Requester strategy $\mathcal{R}$ and a Supplier strategy $\mathcal{S}$ on a graph $G$ are fixed, the sequence of moves is fully determined. We refer to this sequence as the $(\mathcal{R}, \mathcal{S})$-game.

Lemma 5.6. For a fixed vertex $v$ in a graph $G$, Supplier has an optimal strategy $\mathcal{S}$ for the game on $G$ having the following two properties:

(1) $\mathcal{S}$ always provides the same color $c_{1}$ at $v$ in response to the first request made at $v$, regardless of what earlier requests have already been made.

(2) For any Requester strategy $\mathcal{R}$, if there are $d(v)+1+q$ requests at $v$ in the $(\mathcal{R}, \mathcal{S})$-game, where $q \geq 0$, then the total number of requests in the $(\mathcal{R}, \mathcal{S})$-game is at least $\chi_{\mathrm{ISC}}(G)+q$. Furthermore, all colors supplied at $v$ by $\mathcal{S}$ after the first $d(v)$ colors can be chosen arbitrarily, as long as they have not yet been supplied at $v$.

Proof. Let $\mathcal{S}^{\prime}$ be an optimal Supplier strategy. We define $\mathcal{S}$ in terms of $\mathcal{S}^{\prime}$. First, let $c_{1}$ be the color that $\mathcal{S}^{\prime}$ would supply at $v$ if Requester made the first request of the game at $v$. Under $\mathcal{S}$, Supplier pretends that this move has been made against $\mathcal{S}^{\prime}$ and continues playing according to $\mathcal{S}^{\prime}$ as if $c_{1}$ has already been supplied at $v$, until the first actual request is made at $v$. Strategy $\mathcal{S}$ then actually supplies $c_{1}$ at $v$, but pretends that no request was actually made then, since in the imagined game played by $\mathcal{S}^{\prime}$ that request came earlier. This point must be reached, because the actual game cannot end without a request being made at $v$. 
Strategy $\mathcal{S}$ then continues playing the game according to $\mathcal{S}^{\prime}$ (as long as at most $d(v)$ requests have been made at $v$ ). This can be done since the lists $L(w)$ under the actual game played by $\mathcal{S}$ and the imagined game played by $\mathcal{S}^{\prime}$ are now the same for all $w \in V(G)$.

Strategy $\mathcal{S}$ may also deviate from $\mathcal{S}^{\prime}$ when more than $d(v)$ requests are made at $v$. When Requester brings the list size at $v$ to $d(v)+1$, let $c^{\prime}$ be the color that $\mathcal{S}^{\prime}$ would supply. Strategy $\mathcal{S}$ ignores this and instead supplies any color $\hat{c}$ not already used at $v$, playing the subsequent game as if $c^{\prime}$ had actually been supplied. In response to requests beyond $d(v)+1$, $\mathcal{S}$ supplies any colors not previously supplied at $v$ ("free colors"), and for other moves $\mathcal{S}$ plays $\mathcal{S}^{\prime}$ as if the requests beyond $d(v)+1$ requests at $v$ have not occurred.

The game continues until a proper coloring $\phi$ can be chosen from the lists. When this occurs, colors supplied at $v$ after the first $d(v)+1$ requests can be ignored, since $\phi$ can be chosen without using them (because at most $d(v)$ colors occur on neighbors of $v$ ). Also, if $\phi$ requires using $\hat{c}$ on $v$, then the first $d(v)$ colors supplied by $\mathcal{S}^{\prime}$ must be used by $\phi$ on the neighbors of $v$. This means that in the imagined game played according to $\mathcal{S}^{\prime}$, the color $c^{\prime}$ at $v$ completes a proper coloring $\phi^{\prime}$. Since $\mathcal{S}^{\prime}$ is optimal, at least $\chi_{\mathrm{ISC}}(G)$ requests must have been made in addition to the $k-d(v)-1$ made beyond the first $d(v)+1$ requests at $v$.

Lemma 5.7. If $r+1$ is triangular, then $\chi_{\mathrm{ISC}}(T) \geq \chi_{\mathrm{ISC}}(T-R)+r+u_{r}$.

Proof. The degree of $v$ in $T-R$ is 1. Lemma 5.6 allows us to fix an Supplier strategy $\mathcal{S}$ on $T-R$ such that, if Requester makes $2+q$ requests at $v$, where $q \geq 0$, then the total number of requests made on $T-R$ is at least $\chi_{\mathrm{ISC}}(T-R)+q$. Furthermore, Lemma 5.6 guarantees that $\mathcal{S}$ can fix the sequence of colors to be supplied at $v$ (in the game on $T-R$ ) independently of what Requester does, so that the same colors are always supplied at $v$ in the same order. Let $c_{1}, \ldots, c_{u_{r}+1}$ be the first $u_{r}+1$ colors planned by Supplier to be supplied to $v$, independently of the Requester strategy $\mathcal{R}$.

We extend $\mathcal{S}$ to a strategy on all of $T$ by specifying an initial color $\alpha(x)$ for each $x \in R$, to be supplied in response to the first request at $x$, and thereafter supplying arbitrary colors at $x$ in response to further requests. To specify $\alpha$, note that $r=-1+\sum_{i=1}^{u_{r}+1}\left(u_{r}+2-i\right)$, since $r+1$ is triangular and $u_{r}=\max \left\{k:\left(\begin{array}{c}k+1 \\ 2\end{array}\right) \leq r\right\}$. Supplier gives initial color $c_{1}$ to $u_{r}$ vertices in $R$. For $2 \leq i \leq u_{r}+1$, Supplier gives initial color $c_{i}$ to $u_{r}-i+2$ vertices in $R$.

We claim that $\mathcal{S}$ forces Requester to make at least $\chi_{\mathrm{ISC}}(T-R)+r+u_{r}$ requests. Let $\mathcal{R}$ be an optimal strategy for Requester, and let $c$ be the color given to $v$ in a proper coloring chosen from the lists when the game ends.

If $c \notin\left\{c_{1}, \ldots, c_{u_{r}+1}\right\}$, then Requester has made at least $u_{r}+2$ requests at $v$. Hence Requester has made at least $\chi_{\mathrm{ISC}}(T-R)+u_{r}$ requests on $T-R$. Together with the $r$ initial requests at $R$, at least $r+u_{r}+\chi_{\mathrm{ISC}}(T-R)$ requests have been made, as desired.

If $c=c_{i}$ for some $i$, then Requester has made at least $i$ requests at $v$ plus an extra request at each $x \in R$ such that $\alpha(x)=c_{i}$. If $i=1$, then explicitly this counts at least $r+u_{r}+\chi_{\mathrm{ISC}}(T-R)$ requests. If $i \geq 2$, then Requester has made at least $\chi_{\mathrm{ISC}}(T-R)+i-2$ requests on $T-R$ and $r+u_{r}-i+2$ requests on $R$, again at least $r+u_{r}+\chi_{\mathrm{ISC}}(T-R)$.

Lemmas 5.4, 5.5, and 5.7 complete the proof of Theorem 5.1. We note that the arguments can be modified to handle also the case of stars. 


\section{References}

[1] A. Berliner, U. Bostelmann, R. A. Brualdi, and L. Deaett, Sum list coloring graphs, Graphs Combin. 22 (2006), no. 2, 173-183. MR 2231989 (2007c:05070)

[2] M. Bonamy and K. Meeks, The interactive sum choice number of graphs, 2017, arXiv: 1703.05380.

[3] J. M. Carraher, T. Mahoney, G. J. Puleo, and D. B. West, Sum-paintability of generalized theta-graphs, Graphs and Combinatorics 31 (2015), 1325-1334.

[4] P. Erdős, A. L. Rubin, and H. Taylor, Choosability in graphs, Proceedings of the West Coast Conference on Combinatorics, Graph Theory and Computing (Humboldt State Univ., Arcata, Calif., 1979) (Winnipeg, Man.), Congress. Numer., XXVI, Utilitas Math., 1980, pp. 125-157. MR 593902 (82f:05038)

[5] G. Gutowski, T. Krawczyk, D.B. West, M. Zajac, and X. Zhu, Upper bounds for sumcolor cost of outerplanar, planar and $k$-degenerate graphs, preprint.

[6] B. Heinold, Sum choice numbers of some graphs, Discrete Math. 309 (2009), no. 8, 2166-2173. MR 2510342 (2010m:05278)

[7] B. Heinold, The sum choice number of $P_{3} \square P_{n}$, Discrete Appl. Math. 160 (2012), no. 78, 1126-1136. MR 2901131

[8] G. Isaak, Sum list coloring $2 \times n$ arrays, Electron. J. Combin. 9 (2002), no. 1, Note 8, 7. MR 1928792 (2003j:05046)

[9] G. Isaak, Sum list coloring block graphs, Graphs Combin. 20 (2004), no. 4, 499-506. MR 2108395 (2005h:05077)

[10] T. Mahoney, G. Puleo, and D.B. West, Online sum-paintability: The slow-coloring game on graphs, 2015, arXiv:1507.06513.

[11] T. Mahoney, C. Tomlinson, and J. Wise, Families of online sum-choice-greedy graphs, Graphs and Combinatorics 31 (2015), 2309-2317.

[12] U. Schauz, Mr. Paint and Mrs. Correct, Electron. J. Combin. 16 (2009), no. 1, Research Paper 77, 18. MR 2515754 (2010i:91064)

[13] V. G. Vizing, Coloring the vertices of a graph in prescribed colors, Diskret. Analiz (1976), no. 29 Metody Diskret. Anal. v Teorii Kodov i Shem, 3-10, 101. MR 0498216 (58 \#16371)

[14] X. Zhu, On-line list colouring of graphs, Electron. J. Combin. 16 (2009), no. 1, Research Paper 127, 16. MR 2558264 (2011a:05123) 